\title{
Multikinaseinhibitor: Lebensqualität steigt unter langfristiger Therapie
}

— Bei Patienten mit Nierenzellkarzinom kann es bei der Therapie mit einem Multikinaseinhibitor zu Beginn ein „Tal der Tränen" geben. Doch die Toxizität geht rasch zurück. Die Zahl der für die Lebensqualität relevanten Tage ohne Symptome und Toxizität ist hoch.

Prof. Elke Jäger, Frankfurt/Main, berichtete über eine aktuelle Studie zur Lebensqualität von Patienten mit metastasiertem Nierenzellkarzinom unter Therapie mit dem Multikinaseinhibitor Sunitinib (Sutent ${ }^{\circledR}$ ). Die Daten waren zuvor beim Kongress der American Society of Oncology (ASCO 2010) vorgestellt worden.

Untersucht wurde anhand von Daten einer Phase-III-Studie, in der Sunitinib mit Interferon alpha verglichen wurde, inwieweit sich Toxizität und das toxizitätsadjustierte, progressionsfreie Überleben (PFS) bei den beiden Therapien unterscheiden. Beim toxizitätsadjustierten PFS werden die Tage ohne Symptome und Toxizität gezählt. „Die Untersuchung zeigte wie erwartet, dass es bei Therapie mit Sunitinib mehr Tage mit Grad 3/4-Toxizitäten gab als bei der Interferontherapie“, so Jäger. Schauten sich die Wissenschaftler allerdings die Tage ohne Progression und Toxizität an, sah das Ergebnis ganz anders aus. Bei Therapie mit Sunitinib waren es im Median 335 Tage, bei der Interferon-Therapie nur 172.

In diesem Unterschied spiegelt sich das wesentlich längere progressionsfreie Überleben bei Therapie mit Sunitinib wider. Die Zahlen seien aber auch ein Beleg dafür, dass die Toxizität unter Sunitinib im Wesentlichen ein Phänomen der ersten Therapiewochen ist. ,Die kumulative Toxizität ist bei Sunitinib vergleichsweise ge- ring. Darin unterscheidet sich die Substanz von vielen klassischen Tumortherapien“, betonte Jäger.

Diese Zusammenhänge zu kennen, sei wichtig, um die Patienten gut zu führen, betonte Jäger in Berlin. Die Patienten sollten auf mögliche unerwünschte Wirkungen zu Beginn der Therapie hingewiesen werden, aber auch darauf, dass die Lebensqualität im Verlauf der Behandlung dann deutlich steige. ,Damit erhöhen wir die Chancen auf eine gute Compliance“, erläuterte Jäger.

gvg

Post-ASCO-Pressegespräch „Zielgerichtete Therapie beim metastasierten Nierenzellkarzinom/Erfolge und Perspektiven im Spiegel des ASCO 2010“, Berlin, 29. Juni 2010.

Veranstalter: Wyeth/Pfizer Oncology, Berlin

\section{Bei Nykturie die nächtliche Polyurie behandeln}

- Die Nykturie - definiert als eine oder mehr Miktionen pro Nacht - ist die bei Männern und Frauen die häufigste Störung aus dem Bereich LUTS (lower urinary tract symptoms). Die Fragmentierung des Schlafes beeinträchtigt Lebensqualität, Leistungsfähigkeit, Allgemeinbefinden

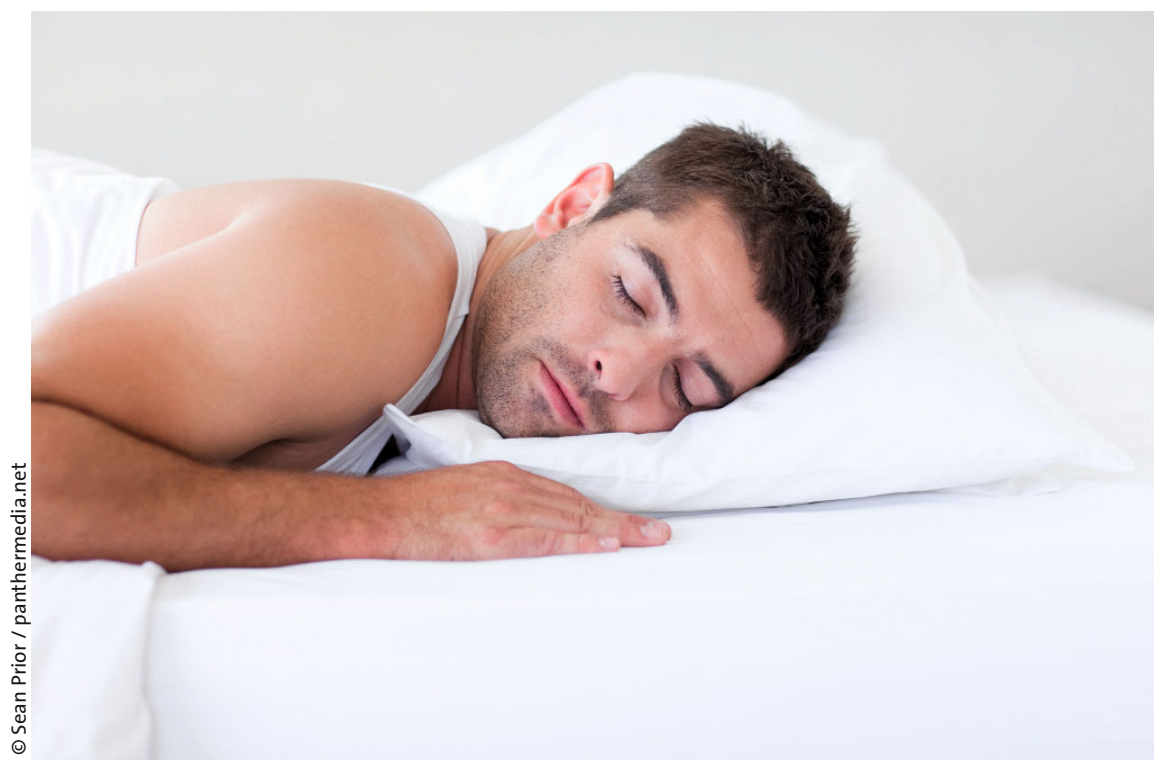

Nykturietherapie - weniger nächtliche Toilettengänge sorgen für mehr Lebensqualität. sowie die psychische und physische Gesundheit der Betroffenen. Bei bis zu $80 \%$ der Patienten liegt der Nykturie eine pathologisch gestörte Urinproduktion in der Nacht zugrunde. Therapieansätze, die sich gegen eine überaktive Blase (OAB) oder eine benigne Prostatahyperplasie (BPH) 